\title{
Polarization and Optical Excitation Function of Resonance Radiation of Cadmium Atoms Excited by Electron Impact
}

\author{
D. Dziczek, M. Piwiński, M. Grądziel and S. Chwirot \\ Institute of Physics, Nicholas Copernicus University \\ Grudziądzka 5/7, 87-100 Toruń, Poland
}

(Received May 31, 2002; revised version October 14, 2002)

\begin{abstract}
First measurements of polarization of $228.8 \mathrm{~nm}$ radiation emitted by cadmium atoms excited to the first singlet state $\left(5^{1} P_{1}\right)$ following impact of electrons were carried out for the electron energy range $20-500 \mathrm{eV}$. New data were also obtained on the relative optical excitation function up to electron energy of $500 \mathrm{eV}$, which extend lower energy results published previously by other researchers.
\end{abstract}

PACS numbers: 34.80.Dp

\section{Introduction}

Cadmium belongs, with zinc and mercury, to the group of elements with two $s$ valence electrons added to the filled $d$ shell. Relatively strong intercombination lines observed in the spectra of these elements prove the spin-orbit interaction to be of comparable or higher strength than the electrostatic one. Fairly heavy Cd atom (roughly the mid-way between well-studied $\mathrm{He}$ and $\mathrm{Hg}$ ) is an interesting target for studies of the departure from $L S$ towards $j j$ coupling scheme of electronic orbital and spin angular momenta with increasing atomic mass. Electron scattering experiments can also provide information on relative significance of spin-flip and electron exchange collisions.

The body of accumulated experimental data on electron-cadmium atom collisions is quite large. Zapesochnyi and Palinchak reported the first measurements 
of the apparent excitation function of the $228.8 \mathrm{~nm}$ resonance line (electron energy range from threshold to $100 \mathrm{eV}$ ) in 1966 [1]. In the same year Zapesochnyi and Shpenik [2] presented high-resolution threshold and low energy study (up to $15 \mathrm{eV})$ of the excitation to two lowest triplet states $\left(5^{3} P_{1}\right.$ and $\left.6{ }^{3} P_{1}\right)$. Similar measurements for singlet states $\left(5^{1} P_{1}, 7^{1} S_{0}, 8^{1} S_{0}\right)$ and for triplet $D$ states $\left(5^{3} D_{1,2,3}\right.$ and $\left.6{ }^{3} D_{1,2,3}\right)$ were published by Shpenik et al. in 1973 [3]. Excitation to $5{ }^{3} P_{0}$ metastable state has been studied by Mazing et al. [4]. Data on excitation functions up to $15 \mathrm{eV}$ for 26 transitions were summarized by Sovter et al. [5]. Results of measurements for a set of atomic and ionic lines of cadmium up to $150 \mathrm{eV}$ were also reported by Bogdanova et al. [6].

Electron energy loss spectra studies on cadmium atoms, yielding data on the differential cross-section, have started from forward-scattering measurements of Newell et al. [7] for electron impact-induced transitions between the ground $5{ }^{1} S_{0}$ and a number of excited states at the incident electron energies: 60, 75, 85, 100, and $150 \mathrm{eV}$. In 1972 Newell and Ross [8] reported measurements of differential cross-sections and data on generalized oscillator strength at $255 \mathrm{eV}$ for the resonance transition and scattering angles between $3^{\circ}$ and $20^{\circ}$. Electron impact excitation of autoionizing levels of $\mathrm{Cd}$ atoms was studied by the same researchers in high (Wickes et al. [9]) and low energy range (Newell et al. [10]). Measurements of the elastic differential cross-section at energies of $60,75,85,100$, and $150 \mathrm{eV}$ in the scattering angle range from $10^{\circ}$ to $70^{\circ}$ were reported by Nogueira et al. [11]. Results of a very extensive study of elastic and inelastic scattering of electrons by cadmium atoms were published by Marinković et al. [12]. Differential cross-sections data for elastic scattering and excitation to a number of states at incident electron energies of $3.4,6.4,10,15,20,40,60$, and $85 \mathrm{eV}$ in the angular range from $0^{\circ}$ to $150^{\circ}$ were presented.

The impressive progress of experimental methods in the field of scattering of polarized electrons from atomic targets contributed to quantitative understanding of the role of different spin-dependent interactions in electron-atom collisions. As mentioned, cadmium is a very interesting target from this point of view. Results of studies on scattering of polarized electrons by cadmium atoms were reported by Bartsch et al. [13].

Despite some serious technical problems connected with long-term and stable operation of efficient sources of beams of cadmium atoms, this element has been one of more popular subjects of electron scattering experiments for many years. Methods for theoretical description of inelastic collisions of charged particles with atoms and molecules were developed concurrently. Reformulation of the Bethe theory by Inokuti [14], Inokuti et al. [15] with further methodological developments introduced by Haffad et al. [16], Avdonina et al. [17] provided researchers with tools useful for normalization of differential cross-section by means of generalized oscillator strengths. Those methods were applied in case of electron-cadmium collisions: Newell et al. [7], Newell and Ross [8], Felfli and Msezane [18]. The distorted-wave 
approximation methods have been used for $a b$ initio calculations of the differential cross-sections for elastic and inelastic electron scattering on cadmium atoms. The first order distorted wave Born approximation (DWB1) results of Madison et al. [19] showed very good agreement with experimental data of Marinković et al. [12], except some localized discrepancies for the ${ }^{3} P$ states. Subsequent calculations of Srivastava et al. [20] applying completely relativistic distorted-wave theory improved the agreement with experimental results.

Despite the extensiveness of the set of data on cadmium accumulated so far by many researchers, it lacks experimental results for the polarization of the radiation and for the excitation function in the range of moderate and higher electron energy even for the resonant transition. Such data have been obtained in the present work.

\section{Experimental method}

\subsection{Apparatus}

Figure 1 presents a schematic of the experimental setup used in measurements of polarization of the $228.8 \mathrm{~nm}$ radiation emitted by cadmium atoms excited by electron impact. Electron-atom collisions were studied in the standard cross-beam configuration. Atomic beam of $\mathrm{Cd}$, produced by resistively heated oven, effused vertically upwards from a system of beam-shaping apertures. The temperature of the reservoir of cadmium was stabilized in the range 550-554 $\mathrm{K}$ with the output nozzle kept at temperature $30 \mathrm{~K}$ higher to slow down clogging of its orifice. The beam-shaping apertures were chilled by water circulating in a cooling jacket

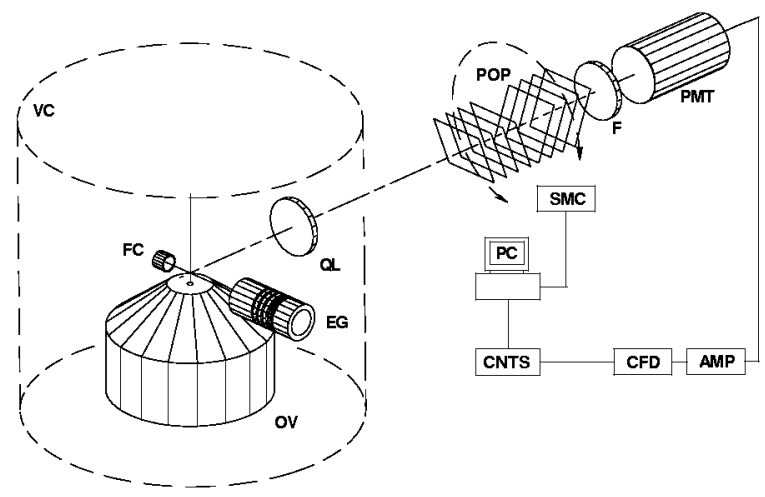

Fig. 1. Schematic of the experimental setup for polarization measurements. EG electron gun, FC - Faraday cup, OV - the source of cadmium atomic beam, QL quartz lens, POP - pile-of-plates polarizer, F - $228.8 \mathrm{~nm}$ interference filter, PMT photomultiplier, AMP - preamplifier, CFD - discriminator, SMC - stepper motor controller, CNTS - pulse counters, VC — vacuum chamber with magnetic shield, PC - microcomputer. 
surrounding all hot parts of the oven, preventing the heating of the other elements of the system by thermal radiation. To avoid deposition of cadmium on surfaces inside the vacuum chamber the beam was captured by a liquid nitrogen cold trap, positioned directly above the source. With the vacuum chamber background pressure maintained at $1 \times 10^{-6}$ Torr, the estimated concentration of cadmium in the region of interaction with electrons was $10^{11}$ atoms $/ \mathrm{ccm}$. The diameter of the cross-section of slightly diverging atomic beam at the level of the interaction region was approximately $2 \mathrm{~mm}$. The electron beam produced by a Comstock EG-402EL electron gun was directed horizontally. The electron beam current, collected by a Faraday cup, was in the range from $1.3 \mu \mathrm{A}$ to $13.3 \mu \mathrm{A}$ depending on electron energy. The beam was focused with gun's electrostatic lens to maintain constant overlap volume with the atomic beam.

The radiation emitted by electron impact-excited Cd atoms was detected in the direction perpendicular to both atomic and electron beams. A fused silica lens focused onto the electron-atom interaction region (acceptance angle $0.19 \mathrm{rad}$ ) was used to form a collimated beam of radiation leaving the vacuum chamber. A pile-of-plate polarizer consisting of two sets of 4 fused silica plates was used as the linear polarization analyzer. The angle of incidence of the radiation at the plates was optimized for the best trade-off between the polarizance and transmission of the detected ultraviolet light. The measured value of the polarizance at $228.8 \mathrm{~nm}$ was $0.786 \pm 0.009$. The polarizer was rotated around the axis of the light beam with a stepper motor controlled by a microcomputer, and the orientation of its polarization axis relative to the direction of the electron beam was changed by data acquisition software. The radiation emerging from the analyzer and transmitted by a $228.8 \mathrm{~nm}$ interference filter was detected by a photomultiplier tube operated in the photon counting mode.

The vacuum collision chamber was lined with a $\mu$-metal shield to attenuate stray external magnetic fields and all sources of electric fields were carefully shielded with grounded copper enclosures and braids to minimize the influence on electron trajectories and collision processes.

The same experimental setup was used for determination of the relative optical excitation function, except the polarization analyzer, which was removed from the light path to allow measurements of the total intensity of the $228.8 \mathrm{~nm}$ emission of cadmium atoms.

\subsection{Procedures}

\subsubsection{Determination of the polarization}

The linear polarization $P$ of the $228.8 \mathrm{~nm}$ radiation emitted by cadmium atoms in direction perpendicular to the electron beam axis (symmetry axis of the collision system) was determined as

$$
P=\frac{I_{\|}-I_{\perp}}{I_{\|}+I_{\perp}},
$$


where $I_{\|}$and $I_{\perp}$ are intensities of the radiation polarized in plane parallel and perpendicular to the direction of the electron beam, respectively. Their values were determined by a set of measurements of the intensity of the radiation transmitted by the polarization analyzer at 28 different positions of its axis (covering the $180^{\circ}$ range of the angle between the polarizing axis and the plane of the electron and light beams). A model function was fitted to the 28 data points yielding the precise values of $I_{\|}$and $I_{\perp}$, and the value of the measured linear polarization degree $P_{\mathrm{m}}$ was calculated using formula (1). Three factors were taken into account to determine the actual value of $P$ : the angular divergence of the focused electron beam, detection of the radiation emitted in a solid angle rather than strictly in one direction and the value of polarizance of the pile-of-plates polarizer lower than 1. Each of the three factors causes that the observed polarization is lower than the actual one. The relation between $P$ and $P_{\mathrm{m}}$ accounting for these effects, as outlined in details in Ehlers and Gallagher [21], is

$$
P_{\mathrm{m}}=\frac{k_{\|}+k_{\perp}}{k_{\|}-k_{\perp}} \frac{1-\varepsilon}{1-\varepsilon P} \frac{1-3 \delta}{1-\delta P} P,
$$

where $k_{\|}$and $k_{\perp}$ are intensity transmission coefficients of the polarizer for the radiation polarized parallel and perpendicular to the optical axis, respectively; $\varepsilon=\psi^{2} / 4, \psi$ - the half-angle of the cone of acceptance of the photon detection channel; $\delta=\theta^{2} / 4, \theta$ - angular divergence of the electron beam (half-angle). The values of the parameters used to calculate the final corrected values of $P$ presented in this work: polarizance of the pile-of-plate analyzer $\left(k_{\|}-k_{\perp}\right) /\left(k_{\|}+k_{\perp}\right)$ $=0.786 \pm 0.009, \psi=0.19 \mathrm{rad}$ and $\theta=0.125 \mathrm{rad}$.

\subsubsection{Determination of the excitation function}

The relative optical excitation function describes the dependence of the total intensity $I_{\text {total }}$ of the radiation emitted by atoms excited to a given state by electron impact on the energy of projectiles. Due to the anisotropy of dipole emission of the atomic system excited in a collision processes of the axial symmetry, the observed radiation intensity changes with the angle between the direction of observation and the symmetry axis. Since the dependence of the intensity on the observation angle is known precisely (Percival and Seaton [22]), $I_{\text {total }}$ can be extracted from a measurement of the intensity at any angle (except $0^{\circ}$ ). In the case of detection of photons emitted perpendicularly to the electron beam (as in our experiment)

$$
I_{\text {total }}=4 \pi I\left(90^{\circ}\right)(1-P / 3)
$$

where $I\left(90^{\circ}\right)$ denotes observed intensity normalized to the unit solid angle, and $P$ is the degree of linear polarization of the radiation emitted in a direction perpendicular to the symmetry axis of the collision system. Accounting for the divergence of the electron beam and finite acceptance angle of the radiation detection system introduces an additional correction factor (Ehlers and Gallagher [21]) yielding final formula used in calculation of values of the total intensity 


$$
I_{\text {total }}=4 \pi I\left(90^{\circ}\right)(1-P / 3) /[1-(\varepsilon+\delta) P]
$$

with $\varepsilon$ and $\delta$ defined as in Eq. (2) and of the same values. The relative optical excitation function $R(E)$ was determined by application of the following formula:

$$
R(E)=\frac{I_{\text {total }}(E)}{J(E)} \frac{J\left(E_{\mathrm{r}}\right)}{I_{\text {total }}\left(E_{\mathrm{r}}\right)}
$$

which normalized the values of the total intensity $I_{\text {total }}(E)$ for a given electron energy $E$ to unit electron beam current and related them to the value determined at a preselected reference electron energy $E_{\mathrm{r}} . J(E)$ is the electron beam current at given energy $E$. Each measurement of the radiation intensity and the electron current for all studied values of energy was followed by determination of these two parameters at the reference energy. All four results were used to calculate the value of $R(E)$ according to Eq. (5). This procedure accounted for the effects of long term fluctuations in the electron beam current and the density of the atomic beam.

Both experiments were carried out at a relatively low density of cadmium atoms in the interaction region $\left(10^{11}\right.$ atoms $\left./ \mathrm{ccm}\right)$. No indication of resonance trapping has been found at this density in a test measurement of the dependence of linear polarization of $228.8 \mathrm{~nm}$ emission on the temperature of the oven.

\section{Results}

\subsection{Polarization}

The degree of linear polarization $P$ of the radiation of wavelength $228.8 \mathrm{~nm}$ emitted by cadmium atoms excited by electron impact to the first singlet state $5{ }^{1} P_{1}$ were measured for 18 values of electron energy in the range from $20 \mathrm{eV}$ to $500 \mathrm{eV}$. Data are plotted in Fig. 2 and listed in Table. Statistical uncertainties of

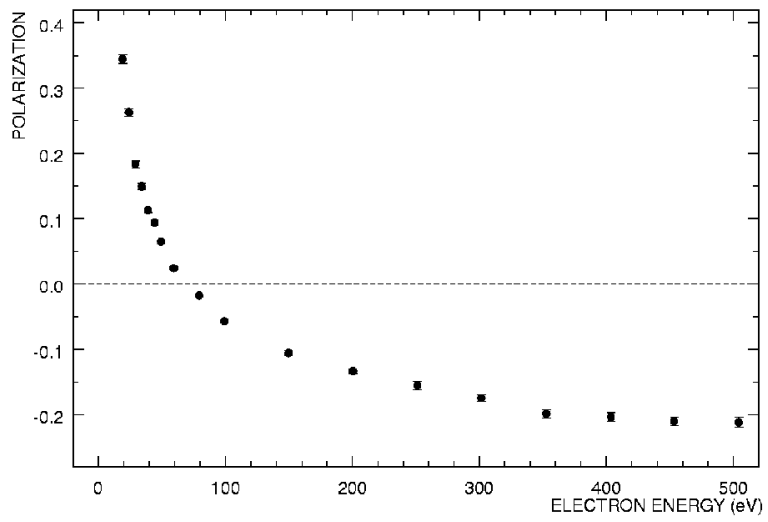

Fig. 2. Measured values of polarization of $228.8 \mathrm{~nm}$ radiation of cadmium atoms excited to $5{ }^{1} P_{1}$ state by electron impact. Error bars represent values of one standard deviation. 
TABLE

Measured values of polarization of $228.8 \mathrm{~nm}$ radiation emitted by cadmium atoms excited to $5^{1} P_{1}$ state by electron impact. Statistical uncertainty of the results (included in brackets) is represented by estimated value of one standard deviation.

\begin{tabular}{c|c||c|c}
\hline \hline Energy (eV) & Polarization & Energy (eV) & Polarization \\
\hline 19 & $0.345(0.007)$ & 99 & $-0.057(0.002)$ \\
24 & $0.263(0.006)$ & 149 & $-0.105(0.004)$ \\
29 & $0.184(0.005)$ & 200 & $-0.133(0.004)$ \\
34 & $0.150(0.005)$ & 251 & $-0.155(0.006)$ \\
39 & $0.113(0.004)$ & 302 & $-0.175(0.006)$ \\
44 & $0.094(0.004)$ & 353 & $-0.199(0.006)$ \\
49 & $0.065(0.003)$ & 404 & $-0.204(0.007)$ \\
59 & $0.025(0.002)$ & 453 & $-0.210(0.006)$ \\
79 & $-0.018(0.002)$ & 504 & $-0.212(0.008)$
\end{tabular}

the present measurements (included in brackets in Table and as error bars in the figure) represent estimated values of one standard deviation.

\subsection{Excitation function}

Values of the relative optical excitation function of the $5{ }^{1} P_{1}$ state of cadmium atoms excited by electron impact were determined for 15 values of electron energy in the range from $35 \mathrm{eV}$ to $500 \mathrm{eV}$. Data are plotted in Fig. 3. Results of previous measurements of Zapesochnyi and Palinchak [11] are also included in the

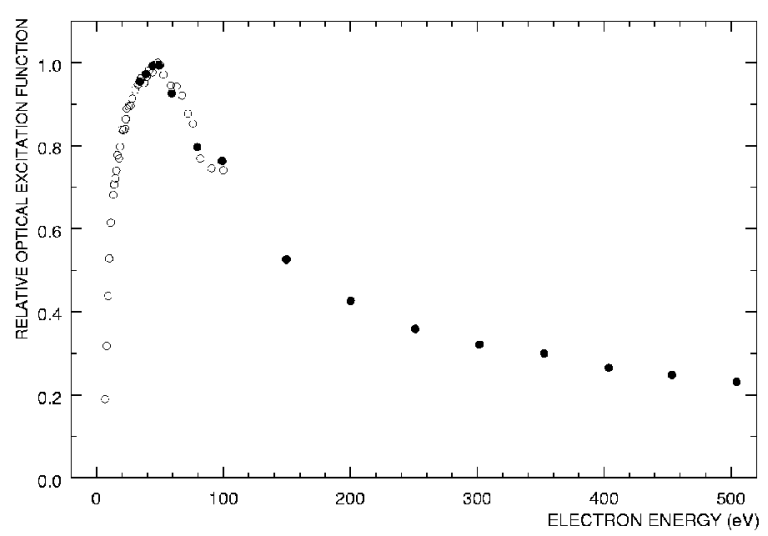

Fig. 3. Measured values of the relative optical excitation function for the $5^{1} P_{1}$ state of cadmium atoms excited by electron impact (• - present work, o — data of Zapesochnyi and Palinchak [1]). 
figure. Both sets were normalized to unity in the area of the maximum of the excitation function. Estimated statistical uncertainties of the present measurements are represented by the size of the data point symbols.

\section{Conclusions}

Polarization of the radiation of cadmium atoms excited to the first singlet state by collisions with electrons has been measured for the first time. New data on the relative optical excitation function have been also obtained, which extend previously published sets of low energy results of Shpenik et al. [3] and intermediate energy measurements of Zapesochnyi and Palinchak [1] up to the energy of $500 \mathrm{eV}$.

Reliable estimation of the effects of the cascade transitions, which may be significant in the case of cadmium, on the observed excitation function and polarization has not been possible. To the best of our knowledge, available quantitative data on the cross-sections for the electron impact excitation of higher levels are not sufficient to determine the cascade contribution to the population of $5^{1} P_{1}$ state. No theoretical predictions for the value of polarization at the energy of excitation threshold for the $5{ }^{1} P_{1}$ state $(5.41 \mathrm{eV})$ are available, which could allow estimation of fractions of the population corresponding to direct and cascade excitation processes based on Bethe approximation using methods outlined by Heddle [23].

\section{Acknowledgments}

The authors acknowledge the support of the State Committee for Scientific Research (grant no. 2 P03B 021 18).

\section{References}

[1] I.P. Zapesochnyi, Y.V. Palinchak, Ukr. Fiz. Zh. (Russ. Ed.) 11, 775 (1966).

[2] I.P. Zapesochnyi, O.B. Shpenik, Zh. Eksp. Teor. Fiz. 50, 890 (1966).

[3] O.B. Shpenik, I.P. Zapesochnyi, V.V.Sovter, E.E. Kontrosh, A.N. Zavilopulo, Zh. Eksp. Teor. Fiz. 65, 1797 (1973).

[4] M.A. Mazing, V.I. Rakhovskij, G.I. Stotskij, V.M. Shustrikov, Opt. Spektrosk. 37, 464 (1974).

[5] V.V. Sovter, I.P. Zapesochnyi, O.B. Shpenik, Opt. Spektrosk. 36, 826 (1974).

[6] I.P. Bogdanova, S.V. Ryanzantseva, V.E. Yakhontova, Opt. Spektrosk. 45, 651 (1978).

[7] W.R. Newell, K.J. Ross, J.P.B. Wickes, J. Phys. B, At. Mol. Phys. 4, 684 (1971).

[8] W.R. Newell, K.J. Ross, J. Phys. B, At. Mol. Phys. 5, 2304 (1972).

[9] J.P.B. Wickes, K.J. Ross, W.R. Newell, J. Phys. B, At. Mol. Phys. 4, L26 (1971).

[10] W.R. Newell, K.J. Ross, J.P.B. Wickes, J. Phys. B, At. Mol. Phys, 6, L337 (1973). 
[11] J.C. Nogueira, W.R. Newell, W.M. Johnstone, J. Phys. B, At. Mol. Opt. Phys. 20, L537 (1987).

[12] B. Marinković, V. Pejčev, D. Filipović, L. Vušković, J. Phys. B, At. Mol. Opt. Phys. 24, 1817 (1991).

[13] M. Bartsch, H. Geesmann, G.F. Hanne, J. Kessler, J. Phys. B, At. Mol. Phys. 25, 1511 (1992).

[14] M. Inokuti, Rev. Mod. Phys. 43, 297 (1971).

[15] M. Inokuti, Y. Itikawa, J.E. Turner, Rev. Mod. Phys. 50, 23 (1978).

[16] A. Haffad, Z. Felfli, A.Z. Msezane, D. Bessis, Phys. Rev. Lett. 76, 2456 (1996).

[17] N.B. Avdonina, Z. Felfli, A.Z. Msezane, J. Phys. B, At. Mol. Opt. Phys. 30, 2591 (1997).

[18] Z. Felfli, A.Z. Msezane, J. Phys. B, At. Mol. Opt. Phys. 31, L165 (1998).

[19] D.H. Madison, K. Bartschat, R. Srivastava, J. Phys. B, At. Mol. Opt. Phys. 24, 1839 (1991).

[20] R. Srivastava, T. Zuo, R.P. McEachran, A.D. Stauffer, J. Phys. B, At. Mol. Opt. Phys. 25, 1073 (1992).

[21] V.J. Ehlers, A. Gallagher, Phys. Rev. A 7, 1573 (1973).

[22] I.C. Percival, M.J. Seaton, Philos. Trans. R. Soc. Lond. A 251, 113 (1958).

[23] D.W.O. Heddle, J. Phys. B, At. Mol. Opt. Phys. 16, 275 (1983). 